\title{
SINOPSIS DE LAS GRAMÍNEAS DE OAXACA, MÉXICO
}

\author{
Darisol Pacheco Rivera ${ }^{1}$ y Patricia Dávila Aranda ${ }^{2}$ \\ ${ }^{1}$ Universidad del Zulia, Facultad de Agronomía, Departamento de Botánica. \\ Apdo. postal 526, Maracaibo, Edo. Zulia, Venezuela \\ ${ }^{2}$ Universidad Nacional Autónoma de México, Facultad de Estudios Superiores \\ Iztacala, Unidad de Biología, Tecnología y Prototipos (UBIPRO). Av. de los \\ Barrios s/n, Los Reyes, Iztacala, 54090 Tlalnepantla, Edo. de México \\ e-mail: darisolp@hotmail.com y pdavilaa@servidor.unam.mx
}

\begin{abstract}
RESUMEN
Como un aporte al conocimiento de la flora del estado de Oaxaca, México, se realizó una revisión de las gramíneas existentes en dicho estado. Se examinaron especímenes depositados en los principales herbarios nacionales, así como también colectas de ejemplares provenientes de trabajo de campo reciente en la zona. Para la recopilación y organización de la información se elaboró una base de datos. Los resultados obtenidos muestran un total de 141 géneros y 528 especies de esta familia, incluyendo las cultivadas e introducidas, lo que equivale aproximadamente a $68.60 \%$ de los géneros y $46.76 \%$ de las especies presentes en el país. La distribución por subfamilias está representada de la siguiente manera: Centothecoideae ( 2 géneros y 5 especies); Arundinoideae ( 3 géneros y 21 especies); Bambusoideae (16 géneros y 35 especies); Pooideae (34 géneros y 76 especies); Chloridoideae (30 géneros y 141 especies); Panicoideae (56 géneros y 250 especies). Se incluye la lista de todas las especies de gramíneas registradas para el estado de Oaxaca.
\end{abstract}

Palabras clave: México, Oaxaca, pastos, Poaceae.

\begin{abstract}
As a contribution to the knowledge of the flora of the state of Oaxaca, Mexico, a revision of the grasses was undertaken. Herbarium specimens from the main herbaria in Mexico were examined, as well as those recently collected in the region. A data base was created in order to compile and organize the available information. The results obtained show a total of 141 genera and 528 of native, introduced and cultivated species of grasses. These numbers approximately represent $68.60 \%$ of the grass genera and the $46.76 \%$ of the grass species that are present in Mexico. At the subfamily level the distribution of taxa is
\end{abstract}


as follows: Centothecoideae ( 2 genera and 5 species); Arundinoideae ( 3 genera and 21 species); Bambusoideae (16 genera and 35 species); Pooideae (34 genera and 76 species); Chloridoideae (30 genera and 141 species); Panicoideae (56 genera and 250 species). The complete list of all the grass species registered for the state of Oaxaca is included.

Key words: grasses, Mexico, Oaxaca, Poaceae.

\section{INTRODUCCIÓN}

En México, las gramíneas constituyen por su diversidad la tercera familia más importante, precedida por las asteráceas y fabáceas (Valdés y Cabral, 1993). Las estimaciones más recientes señalan entre 198 y 206 géneros y un poco más de 1,000 especies a nivel nacional, representadas en 6 subfamilias, 26 tribus y 30 subtribus (Valdés y Dávila, 1995; Espejo et al., 2000). Los miembros de esta familia han sido estudiados e inventariados por diferentes autores a través de floras regionales y listas florísticas (Beetle, 1975; Beetle et al., 1991; Breedlove, 1986; Dávila et al., 1990, 1993, 1998; González et al., 1991; Gould y Morán, 1981; Herrera, 2001; Lebgue y Valerio, 1991; Martínez, 1998; Melgoza et al., 1988; Rzedowski y Rzedowski, 1990; Sosa et al., 1985; Sosa y Gómez-Pompa, 1994; Téllez, 1995; Valdés, 1977; Vega et al., 1989).

Entre las regiones medianamente exploradas en México se encuentra el estado de Oaxaca, el cual se considera el segundo más rico desde el punto de vista florístico, antecedido por Chiapas, estimándose hasta en 9,000 las especies de plantas superiores presentes en la entidad (Lorence y García, 1989). El conocimiento de esta familia en Oaxaca es aún muy deficiente, por lo que se cree necesario en primer lugar, organizar la gran cantidad de material botánico colectado en diferentes zonas a lo largo de muchos años. El presente estudio intenta contribuir al conocimiento de la flora de Oaxaca, determinando cuántos y cuáles son los géneros y especies de gramíneas (Poaceae) reconocidos para la entidad, así como también evaluar el estado del conocimiento de la familia en México y aportar nuevos datos para el inventario de la flora agrostológica nacional.

\section{ANTECEDENTES}

Se mencionan a continuación algunos trabajos importantes realizados en diferentes regiones de Oaxaca, que permiten tener una idea aproximada del estado 
de conocimiento de la familia Poaceae en dicha entidad. Sousa (1963) enlista 30 especies de gramíneas colectadas en la vegetación secundaria de la región de Tuxtepec, Oaxaca. García-Mendoza (1983), en su estudio florístico de una porción de la Sierra de Tamazulapan, Distrito de Teposcolula, Oaxaca, registra un total de 486 taxa, de los cuales 15 especies corresponden a las gramíneas. Torres (1989) define a las Poaceae dentro de las cinco familias con mayor número de especies, representando $3.33 \%$ del total encontrado para la vegetación del Cerro Guiengola, en el Istmo de Tehuantepec, Oaxaca. Campos (1993) contribuye al conocimiento florístico de la Sierra Madre del Sur, una de las regiones menos exploradas del estado de Oaxaca. Este autor registra 9 géneros y 13 especies de gramíneas en la porción central del municipio de San Jerónimo Coatlán. Dávila et al. (1993) realizan un estudio florístico donde incluyen 79 géneros y 115 especies de Poaceae para el Valle de Tehuacán-Cuicatlán, conformado por partes de los estados de Puebla y Oaxaca. Reyes (1993) en su estudio fitogeográfico y florístico realizado en el municipio de San Juan Mixtepec, distrito de Juxtlahuaca, Oaxaca, ubica a las gramíneas como el tercer grupo en importancia con 34 géneros y 60 especies, mismos que constituyen respectivamente $7.8 \%$ y $7.5 \%$ del total registrado para esta región.

\section{MATERIALES Y MÉTODOS}

Este estudio se realizó con base en la recopilación y sistematización de los datos obtenidos a través de las fases de búsqueda bibliográfica, consulta de herbarios y trabajo de campo. Se revisaron las colecciones de gramíneas para el estado de Oaxaca depositadas en los herbarios nacionales CHAPA, COCA, ENCB, MEXU y OAX (Holmgren et al. 1990). Adicionalmente, se consultó e incorporó el material de reciente colección en la zona proveniente del proyecto Flora de Oaxaca del Instituto de Biología, de la Universidad Nacional Autónoma de México. La tarea de campo no se consideró prioritaria para este trabajo debido a la gran cantidad de material herborizado que necesitaba ser estudiado y, por otra parte, a la extensión y complejidad del estado de Oaxaca.

Todo el material fue determinado hasta el nivel de especie, con la ayuda de floras, monografías, revisiones de la familia, géneros y especies, así como también a través de la consulta a especialistas. La información fue recopilada y sistematizada en una base de datos, con 33 campos de información, utilizando el programa Q\&A versión 4.0. El sistema de clasificación seguido fue el empleado para las gramíneas mexicanas por Valdés y Dávila (1995), adaptado según el esquema de Clayton y Renvoize (1986, 1992), Soderstrom y Ellis (1987), Watson y Dallwitz (1992) y Davidse et al. (1994). Los nombres de los géneros se estandarizaron de acuerdo 
con Clayton y Renvoize (1986) y Watson y Dallwitz (1992). Para la abreviación de los autores se siguió a Brummit y Powell (1992). La lista de los géneros y especies se ordenó alfabéticamente. El nombre de la subfamilia y la localización por distrito de cada una de las especies consideradas, fueron incorporados a esta lista (Apéndice).

\section{RESULTADOS Y DISCUSIÓN}

El estudio florístico realizado de la familia Poaceae para el estado de Oaxaca, indica que el grupo está representado por 6 subfamilias, 22 tribus, 29 subtribus, 141 géneros y cerca de 528 especies (incluyendo las cultivadas e introducidas) (ver Apéndice). Esto representa $84.62 \%$ de las tribus, $96.70 \%$ de las subtribus, $68.60 \%$ de los géneros y $46.76 \%$ de las especies conocidas del país (Valdés y Dávila, 1995). A nivel mundial, constituye $20.29 \%$ y $5.27 \%$ de los géneros y especies respectivamente (según Clayton y Renvoize, 1986). Los 141 géneros y 528 especies de gramíneas, están distribuidas por subfamilias como se indica en el Cuadro 1. Se observa que las Panicoideae y las Pooideae son las más diversas en el estado de Oaxaca, seguidas por las Chloridoideae, Bambusoideae, Arundinoideae y Centothecoideae. Este patrón corresponde con el señalado para el país por Valdés y Cabral (1993), en cuanto a la diversidad de especies por subfamilias.

Comparando los resultados aquí obtenidos, con los encontrados para otras entidades de la República Mexicana (Beetle, 1975; Beetle et al., 1991; Breedlove, 1986; Dávila et al., 1990, 1993, 1998; González et al., 1991; Gould y Morán 1981; Herrera, 2001; Lebgue y Valerio, 1991; Martínez, 1998; Melgoza et al., 1988; Rzedowski y Rzedowski, 1990; Sosa et al., 1985; Sosa y Gómez-Pompa, 1994; Téllez, 1995; Valdés, 1977; Vega et al., 1989), se puede considerar que en el estado actual de conocimiento, Oaxaca es el territorio más diverso en cuanto al número de géneros y especies de gramíneas registradas, seguido por Veracruz, Jalisco, Puebla y Chiapas (Cuadro 2).

Los géneros que resultaron mejor representados para Oaxaca en cuanto al número de especies fueron: Paspalum (41), Muhlenbergia (33), Eragrostis (28), Panicum (26), Bouteloua (22), Aristida (19), Digitaria (18), Setaria (14), Urochloa (12), Andropogon (11), Lasiacis (10), Chusquea, Dichanthelium y Sporobolus (9).

Por otra parte, las especies de gramíneas conocidas hasta el momento sólo para el estado son: Aristida petersonii Allred \& Valdés-Reyna, Arthropogon villosus Nees, Aulonemia fulgor Soderstr., Chusquea aperta L. G. Clark, Chusquea repens L. G. Clark \& Londoño subsp. oaxacensis L. G. Clark \& Londoño, Paspalum ligulare Nees y Mesosetum filifolium F. T. Hubb. Estos taxa 
Cuadro 1. Distribución por subfamilias del número de géneros y especies de gramíneas registradas para Oaxaca.

\begin{tabular}{|l|c|c|}
\hline \multicolumn{1}{|c|}{ Subfamilia } & No. de géneros & No. de especies \\
\hline Centothecoideae & 2 & 5 \\
\hline Arundinoideae & 3 & 21 \\
\hline Bambusoideae & 16 & 35 \\
\hline Chloridoideae & 30 & 141 \\
\hline Pooideae & 34 & 76 \\
\hline Panicoideae & 56 & 250 \\
\hline Total & 141 & 528 \\
\hline
\end{tabular}

se distribuyen en los distritos Centro, Ixtlán, Juchitán, Pochutla y Tuxtepec, en bosques de Quercus, bosques de coníferas, bosques de Quercus-coníferas y pastizales, a un intervalo altitudinal que va desde los 400 hasta los 2,080 m. snm. Las especies mencionadas equivalen a $1.32 \%$ de las registradas para el estado y $0.62 \%$ a nivel nacional.

Es un hecho, que los datos mostrados en el presente trabajo deberán variar en la medida que el conocimiento florístico de Oaxaca y de los demás estados o regiones se complete, pues mientras que para el Valle de México, Valle de Tehuacán-Cuicatlán, Puebla, Durango, Coahuila, Tlaxcala y Baja California, se puede asegurar que la información existente es bastante representativa, el resto de los estados o regiones aquí señalados aún requieren una mayor atención en la recolección y estudio taxonómico de este diverso grupo de plantas. 
Cuadro 2. Número de géneros y especies de gramíneas registrado para diferentes estados o regiones de la República Mexicana.

\begin{tabular}{|c|c|c|c|c|c|}
\hline Estado o región & $\begin{array}{l}\text { No. de } \\
\text { géneros }\end{array}$ & $\begin{array}{c}\% \text { del } \\
\text { total para } \\
\text { el país* }\end{array}$ & $\begin{array}{l}\text { No. de } \\
\text { especies }\end{array}$ & $\begin{array}{c}\% \text { del } \\
\text { total para } \\
\text { el país** }\end{array}$ & Referencia \\
\hline Oaxaca & 141 & 68.1 & 528 & 46.8 & Presente trabajo \\
\hline Veracruz & 124 & 59.9 & 515 & 45.7 & $\begin{array}{l}\text { Sosa y Gómez-Pompa } \\
\text { (1994) }\end{array}$ \\
\hline Chiapas & 123 & 59.4 & 415 & 36.8 & Breedlove (1986) \\
\hline Jalisco & 118 & 57.0 & 430 & 38.1 & Dávila et al. (1998) \\
\hline Puebla & 118 & 57.0 & 427 & 37.9 & Dávila et al. (1990) \\
\hline Chihuahua & 106 & 51.2 & 313 & 27.8 & Lebgue y Valerio (1991) \\
\hline Durango & 97 & 46.9 & 338 & 30.0 & $\begin{array}{l}\text { González et al. (1991); } \\
\text { Herrera (2001) }\end{array}$ \\
\hline $\begin{array}{l}\text { Península de Baja } \\
\text { California }\end{array}$ & 96 & 46.4 & 274 & 24.3 & Gould y Moran (1981) \\
\hline Sonora & 93 & 44.9 & 325 & 28.8 & Beetle et al. (1991) \\
\hline Coahuila & 80 & 38.6 & 295 & 26.2 & Valdés (1977) \\
\hline Nayarit & 80 & 38.6 & 269 & 23.9 & $\begin{array}{l}\text { Melgoza et al. (1988); } \\
\text { Téllez(1995) }\end{array}$ \\
\hline $\begin{array}{l}\text { Valle de Tehuacán- } \\
\text { Cuicatlán }\end{array}$ & 79 & 38.2 & 115 & 10.2 & Dávila et al. (1993) \\
\hline Valle de México & 75 & 36.2 & 262 & 23.2 & $\begin{array}{l}\text { Rzedowski y Rzedowski } \\
\text { (1990) }\end{array}$ \\
\hline $\begin{array}{l}\text { Península de } \\
\text { Yucatán }\end{array}$ & 65 & 31.4 & 183 & 16.2 & Sosa et al. (1985) \\
\hline Tlaxcala & 61 & 29.5 & 182 & 16.1 & Martínez (1998) \\
\hline Colima & 49 & 23.7 & 119 & 10.6 & Beetle (1975) \\
\hline Sinaloa & 49 & 23.7 & 94 & 8.3 & Vega et al. (1989) \\
\hline
\end{tabular}

* Porcentajes calculados con base en 207 géneros (Valdés y Dávila, 1995)

** Porcentajes calculados con base en 1,127 especies (Beetle et al., 1985) 


\section{LITERATURA CITADA}

Beetle, A. A. 1975. Contribución al estudio de las gramíneas de México. Gramíneas de Colima, México. Univ. Wyoming Publ. Edición No. 10. Laramie, Wyoming. 6 pp.

Beetle, A. A., E. Manrique, V. Jaramillo, M. Guerrero, J. A. Miranda, I. Núñez y A. Chimal. 1985. Las gramíneas de México. Vol. 2. Secretaría de Agricultura y Recursos Hidraúlicos. Comisión Técnico Consultiva de Coeficientes de Agostadero. México, D.F. 344 pp.

Beetle, A. A., D. Johnson, A. Navarro y R. Alcaraz. 1991. Gramíneas de Sonora. Secretaría de Agricultura y Recursos Hidraúlicos. Comisión Técnico Consultiva de Coeficientes de Agostadero. Hermosillo, Sonora. 174 pp.

Breedlove, D. E. 1986. Flora de Chiapas. Listados florísticos de México. IV. Instituto de Biología. Universidad Nacional Autonóma de México. México, D.F. 246 pp.

Brummitt, R. K. y C. E. Powell (eds.). 1992. Authors of plant names. Royal Botanical Gardens. Kew. 732 pp.

Campos, V. A. 1993. Estudio florístico de la porción central de San Jerónimo Coatlán (Oaxaca). Tesis de licenciatura. Facultad de Ciencias, Universidad Nacional Autónoma de México. México, D.F. 97 pp.

Clayton, W. D. y S. A. Renvoize. 1986. Genera graminum: Grasses of the world. Kew Bull. Add. Ser. XIII. Royal Botanical Gardens. Kew. 379 pp.

Clayton, W. D. y S. A. Renvoize. 1992. A system of classification for the grasses. In: Chapman, G. P. (ed.). Grass evolution and domestication. Cambridge University Press. Cambridge. pp. 338-353.

Davidse, G., M. Sousa y A. Chater (eds.). 1994. Flora Mesoamericana. Vol. 6. Universidad Nacional Autónoma de México. México, D.F. 543 pp.

Dávila, P., P. Tenorio, E. Manrique, J. A. Miranda y A. Rodríguez. 1990. Listado florístico de las gramíneas de Puebla. Listados florísticos de México VII. Instituto de Biología. Universidad Nacional Autónoma de México. México, D.F. 51 pp.

Dávila, P., J. L. Villaseñor, R. Medina, A. Ramírez, A. Salinas, J. Sánchez-Ken y P. Tenorio. 1993. Poaceae. In: Flora del Valle de Tehuacán-Cuicatlán. Listados florísticos de México X. Instituto de Biología, Universidad Nacional Autonóma de México. México, D. F. pp. 174-184.

Dávila, P., R. Lira, y J. Sánchez Ken. 1998. La familia Gramineae en el estado de Jalisco, México. Boletín IBUG 5(1-3): 191-215.

Espejo, A., A. R. López y J. Valdés-Reyna. 2000. Poaceae. In: Espejo, A y A. R. López. Las monocotiledóneas mexicanas. Una sinopsis florística. 1. Lista de referencia. Partes IX a XI Pandanaceae a Zosteraceae. Consejo Nacional de la Flora de México, Universidad Autónoma Metropolitana Iztapalapa y Comisión Nacional para el Conocimiento y Uso de la Biodiversidad. México, D.F. pp. 8-236.

García-Mendoza, A. 1983. Estudio ecológico-florístico de una porción de la Sierra de Tamazulapan, distrito de Teposcolula, Oaxaca, México. Tesis de licenciatura. Facultad de Ciencias, Universidad Nacional Autónoma de México, México, D.F. $112 \mathrm{pp}$. 
Gould, F. y R. Moran. 1981. The grasses of Baja California, Mexico. Memoir 12. Society of Natural History. San Diego, California. 134 pp.

Herrera, Y. Gramineae. 1991. In: González, E. M., S. González y Y. Herrera. Flora de Durango. Listados florísticos de México IX. Instituto de Biología, Universidad Nacional Autonóma de México. México, D.F. pp. 137-150.

Herrera, Y. 2001. Las gramíneas de Durango. Instituto Politécnico Nacional y Comisión Nacional para el Conocimiento y Uso de la Biodiversidad. México, D.F. 478 pp.

Holmgren, P. K., N. H. Holmgren y L. C. Barnett. 1990. Index Herbariorum. Part. 1: The herbaria of the World. 8 ${ }^{\mathrm{a}}$. ed. New York Botanical Garden. Nueva York. 693 pp.

Lebgue, T., y A. Valerio. 1991. Gramíneas de Chihuahua. Manual de identificación. Universidad Autónoma de Chihuahua. Chihuahua. 301 pp.

Lorence, D. y A. García. 1989. Oaxaca, México. In: Campbell, D. y D. Hammond (eds.). Floristic inventory of tropical countries. New York Botanical Garden. Bronx, Nueva York. pp. 254 - 268.

Manrique, E. A. 1994. The genus Schizachyrium in Mexico and Central America. Ph.D. Thesis. Texas A \& M University, College Station, Texas. 165 pp.

Martínez, J. L. 1998. Flora genérica del estado de Tlaxcala. Tesis de maestría. Universidad Nacional Autónoma de México. México, D. F. 219 pp.

McVaugh, R. 1983. Gramineae. In: Anderson, W. R. (ed.). Flora Novo-Galiciana 14: 1-436. The University of Michigan Press. Ann Arbor.

Melgoza, G., A. Melgoza y A. A. Beetle. 1988. Gramíneas de Nayarit. Univ. Wyoming Pub. Laramie, Wyoming. $33 \mathrm{pp}$.

Reyes, P. J. 1993. Estudio florístico y fitogeográfico en el municipio de San Juan Mixtepec, Distrito de Juxtlahuaca, Oaxaca. Tesis de licenciatura. Escuela Nacional de Estudios Profesionales Iztacala, Universidad Nacional Autónoma de México. Tlalnepantla, Méx. 112 pp.

Rzedowski, J., y G. Calderón de Rzedowski. 1990. Flora fanerogámica del Valle de México. Volumen III. Monocotyledoneae. Instituto de Ecología, Centro Regional del Bajío, Pátzcuaro, Michoacán. 494 pp.

Soderstrom, T. R. y R. P. Ellis. 1987. The position of bamboo genera and allies in a system of grass classification. In: Soderstrom, T. R., K. W. Hilu, C. S. Campbell y M. E. Barkworth (eds.). Grass systematics and evolution. Smithsonian Institution Press. Washington, D.C. pp. 225-238.

Sosa, V., J. Salvador, V. Rico-Gray, R. Lira y J. J. Ortiz. 1985. Gramineae. In: Etnoflora yucatanense. Lista florística y sinonimia maya. Fascículo 1. Instituto Nacional de Investigaciones sobre Recursos Bióticos. Xalapa, Veracruz. pp. 85-89.

Sosa, V. y A. Gómez- Pompa. (comp.). 1994. Gramineae. In: Flora de Veracruz. Lista florística. Fascículo 82. Instituto de Ecología, A. C., Xalapa, Veracruz y University of California, Riverside. pp.109-127.

Sousa S., M. 1963. La vegetación secundaria en la región de Tuxtepec, Oaxaca. Tesis profesional. Facultad de Ciencias. Universidad Nacional Autónoma de México. México, D.F. 63 pp. 
Téllez, O. 1995. Flora, vegetación y fitogeografía de Nayarit, México. Tesis de maestría. Facultad de Ciencias. Universidad Nacional Autonóma de México. México, D.F. 166 pp.

Torres, L. 1989. Estudio floristíco y descripción de la vegetación del Cerro Guiengola, en el Itsmo de Tehuantepec. Tesis de licenciatura. Escuela Nacional de Estudios Profesionales Iztacala. Universidad Nacional Autónoma de México. Tlalnepantla, Méx. $81 \mathrm{pp}$.

Valdés R., J. 1977. Gramíneas de Coahuila. Lista de especies y clave para los géneros. Universidad Autónoma Agraria Antonio Narro. Monografía Técnico Científica. Vol. 3. No. II. Saltillo. pp. 884-1009.

Valdés, R., J. e I. Cabral C. 1993. Chorology of Mexican grasses. In: Ramamoorthy, T. P., R. Bye, A. Lot y J. Fa (eds.). Biological diversity of Mexico, origins and distribution. Oxford University Press. Nueva York. pp. 439-446.

Valdés R., J. y P. Dávila. 1995. Clasificación de los géneros de gramíneas (Poaceae) mexicanas. Acta Bot. Mex. 33: 1-36.

Vega, R., G. Bojórquez y F. Hernández. 1989. Gramineae. In: Flora de Sinaloa. Universidad Autonóma de Sinaloa. Secretaría de Educación Pública Culiacán, Sinaloa. pp. 11-13.

Watson, L. y M. J. Dallwitz. 1992. The grass genera of the world. Center for Agriculture and Biosciences. Wallingford. $1038 \mathrm{pp}$.

Recibido en junio de 2001. Aceptado en octubre de 2004 
Apéndice. Lista de los géneros y especies presentes en el estado de Oaxaca con su respectiva subfamilia y localización por distrito dentro de la entidad. Las abreviaturas utilizadas para designar los 30 distritos del estado son las siguientes: Centro (CEN), Choapan (CHO), Coixtlahuaca (COX), Cuicatlán (CUI), Ejutla (EJU), Etla (ETL), Huajuapam (HUA), Ixtlán (IXT), Jamiltepec (JAM), Juchitán (JUC), Juquila (JUQ), Juxtlahuaca (JUX), Miahuatlán (MIA), Mixe (MIX), Nochixtlán (NOC), Ocotlán (OCO), Pochutla (POC), Putla (PUT), Silacayoapan (SIL), Sola de Vega (SOL), Tehuantepec (TEH), Teotitlán (TEO), Teposcolula (TEP), Tlacolula (TLA), Tlaxiaco (TLX), Tuxtepec (TUX), Villa Alta (VAL), Yautepec (YAU), Zaachila (ZAA) y Zimatlán (ZIM).

Achnaterum P. Beauv.; Pooideae; 2 especies.

1. A. constrictum (Hitchc.) Valdés-Reyna \& Barkworth; (MIX)

2. A. eminens (Cav.) Barkworth; (COX, HUA, TEP)

Acroceras Stapf; Panicoideae; 1 especie.

1. A. zizanioides (Kunth) Dandy; (IXT, TUX)

Aegopogon Willd.; Chloridoideae; 2 especies.

1. A. cenchroides Humb. \& Bonpl. ex Willd.; (CEN, IXT, TEP, VAL)

2. A. tenellus (DC.) Trin.; (HUA, IXT, TEP)

Agrostis L.; Pooideae; 9 especies.

1. A. alba L.; (TLA, VAL)

2. A. ghiesbreghtii E. Fourn.; (CEN, IXT, MIA, MIX, TEP)

3. A. liebmannii (E. Fourn.) A. Hitchc.; registrada por Espejo et al. (2000). No se especifica la localidad dentro del estado.

4. A. perennans (Walter) Tuck.; (IXT, MIA, MIX)

5. A. pittieri Hack.; (TEP)

6. A. scabra Willd.; (IXT, TEP)

7. A. schaffneri E. Fourn.; (CEN)

8. A. subpatens Hitchc.; (JUX)

9. A. virescens Kunth; (CEN, MIA)

Aira L.; Pooideae; 1 especie.

1. A. caryophyllea L.; (IXT)

Andropogon L.; Panicoideae; 11 especies.

1. A. angustatus (J. Presl) Steud.; (JAM, JUC)

2. A. bicornis L.; (JUC, MIX, PUT, TUX)

3. A. bourgaei Hack.; (ETL, TUX)

4. A. fastigiatus Sw.; (JAM, JUC, PUT, TEH)

5. A. gerardii Vitman; (JUC, TUX) 
Apéndice. Continuación.

6. A. glomeratus (Walter) Britton, Stern \& Poggenb.; (CHO, JUX)

7. A. leucostachyus Kunth; (CHO)

8. A. liebmannii Hack.; (JUC, THE)

9. A. pringlei Scribn. \& Merr.; (CEN, CHO)

10. A. selloanus (Hack.) Hack.; (JUC)

11. A. virginicus L.; (IXT)

Anthaenantia P. Beauv.; Panicoideae; 1 especie.

1. A. lanata (Kunth) Benth.; (TEA, IXT)

Anthephora Schreb.; Panicoideae; 1 especie.

1. A. hermaphrodita (L.) Kuntze; (CUI, JUCH, POC, TEH)

Anthoxanthum L.; Pooideae; 2 especies.

1. A. odoratum L.; (JUX)

2. A. mexicanum (Rupr. ex Fourn.) Mez; (CEN, IXT)

Aristida L.; Arundinoideae; 19 especies; dos representadas por variedades y una por forma.

1. A. arizonica Vasey; registrada por Espejo et al. (2000). No se especifica la localidad dentro del estado.

2. A. adscensionis L.; (COX, CUI, HUA, JUC, JUX, TEH, TEO, TEP)

3. A. appressa Vasey; (MIA, TEO)

4. A. divaricata Humb. \& Bonpl. ex Willd; (COX, NOC, TEP)

5. A. eludens Allred \& Valdés-Reyna; registrada por Espejo et al, 2000. No se especifica la localidad dentro del estado.

6. A. gibbosa (Nees) Kunth; (POC)

7. A. hamulosa Henrard; (CEN)

8. A. havardii Vasey; (HUA)

9. A. hintonii A. Hitchc.; registrada por Espejo et al. (2000). No se especifica la localidad dentro del estado.

10. A. hitchcockiana Henrard; registrada por Espejo et al. (2000). No se especifica la localidad dentro del estado.

11. A. jorullensis Kunth; (JUC, PUT)

12. A. laxa Cav. var. longiramea (J. Presl) Henrard; (COX, HUA, NOC, OCO, TEP, TLA)

13. A. liebmannii E. Fourn.; (HUA, TEH, TEO)

14. A. pansa Wooton \& Standl. fo. dissita (I. M. Johnst.) Allred \& Valdés-Reyna; (COX, NOC, TEP)

15. A. petersonii Allred \& Valdés-Reyna; (TLA)

16. A. purpurea Nutt. var. curvifolia (E. Fourn.) Allred; (COX, NOC, POC, TEO, TEP) 
Apéndice. Continuación.

16. A. purpurea Nutt. var. wrightii (Nash) Allred; registrada por Espejo et al. (2000). No se especifica la localidad dentro del estado.

17. A. recurvata Kunth; (PUT)

18. A. schiedeana Trin. \& Rupr.; (COX, CUI, JUX, HUA, NOC, TEO, TEP, TLA)

19. A. ternipes Cav.; (CUI, HUA, NOC, TEO)

Arthraxon P. Beauv.; Panicoideae; 1 especie, representada por una variedad.

1. A. hispidus (Thunb.) Makino var. hispidus; (IXT, TUX)

Arthropogon Ness; Panicoideae; 1 especie.

1. A. villosus Nees; endémica para Oaxaca. Registrada por Davidse et al. (1994) y Espejo et al. (2000). No se especifica la localidad dentro del estado.

Arundinella Raddi; Panicoideae; 3 especies.

1. A. berteroniana (Schult.) A. Hitchc.; (CHO, EJU, JUC, POC, TUX)

2. A. deppeana Nees; (TEH, TUX)

3. A. hispida (Humb. \& Bonpl. ex Willd.) Kuntze; (POC)

Arundo L.; Arundinoideae; 1 especie.

1. A. donax L. (IXT, TEH)

Aulonemia Goudot; Bambusoideae; 2 especies.

1. A. fulgor Soderstr.; (IXT, MIX, TEO, TUX)

2. A. laxa (F. Maek.) McClure; registrada por Espejo et al. (2000). No se especifica la localidad dentro del estado.

Avena L.; Pooideae; 2 especies.

1. A. fatua L.; (HUA, NOC, TEP)

2. A. sativa $\mathrm{L}$.; (CEN, NOC)

Axonopus P. Beauv.; Panicoideae; 5 especies.

1. A. aureus P. Beauv.; (JUC)

2. A. compressus (Sw.) P. Beauv.; registrada por Beetle et al. (1983) y Davidse et al. (1994); no se especifica la localidad dentro del estado.

3. A. poiophyllus Chase; (TUX)

4. A. purpusii (Mez) Chase; (TUX)

5. A. scoparius (Flüggé) Kuhlm.; registrada por Espejo et al. (2000). No se especifica la localidad dentro del estado.

Bambusa Schreb.; Bambusoideae; 1 especie.

1. B. vulgaris Schrad. ex H. Wendl.; (HUA, IXT, JUQ) 
Apéndice. Continuación.

Bothriochloa Kuntze; Panicoideae; 4 especies.

1. B. barbinodis (Lag.) Herter; (CEN, ETL, HUA, IXT, TEH, TEO, TEP)

2. B. hirtifolia (J. Presl) Henr.; (CEN, ETL, NOC, TEO)

3. B. laguroides (DC.) Herter; (ETL, JUX)

4. B. saccharoides (Sw.) Rydb.; (IXT)

Bouteloua Lag.; Chloridoideae; 22 especies.

1. B. alamosana Vasey; registrada por Espejo et al. (2000). No se especifica la localidad dentro del estado.

2. B. aristidoides (Kunth) Griseb.; (CUI, TEO, TEH)

3. B. barbata Lag.; (CUI)

4. B. chondrosoides (Kunth) Benth.; (CEN, COX, HUA. TEH)

5. B. curtipendula (Michx.) Torr.; (CEN, CUI, HUA, TEH, TEO, TEP, TLA)

6. B. dimorpha Columbus; (CEN, CUI, HUA, JUC, OCO)

7. B. distans Swallen; (CUI)

8. B. disticha (Kunth) Benth.; registrada por Espejo et al. (2000). No se especifica la localidad dentro del estado.

9. B. elata J. Reeder \& C. Reeder; (SIL)

10. B. gracilis (Kunth) Lag. ex Griffiths; (IXT, NOC)

11. B. hirsuta Lag.; (CEN, HUA, IXT, TEP, ZIM)

12. B. longiseta Gould; registrada por Espejo et al. (2000). No se especifica la localidad dentro del estado.

13. B. media (E. Fourn.) Gould \& Kapadia; (CUI, JUC, TEH)

14. B. multifida (Griffiths) Columbus; (HUA, NOC, SOL, TEH)

15. B. radicosa (E. Fourn.) Griffiths; (CEN, TEH, TEP)

16. B. reederorum Columbus; (HUA)

17. B. repens (Kunth) Scribn. \& Merr.; (CEN, HUA, TLA)

18. B. scabra (Kunth) Columbus; (JUQ)

19. B. scorpioides Lag.; (COX)

20. B. simplex Lag.; registrada por Espejo et al. (2000). No se especifica la localidad dentro del estado.

21. B. triaena (Trin. ex Spreng.) Scribn.; (CEN, COX, ETL, HUA, TEP)

22. B. williamsii Swallen; (TLX)

Brachypodium P. Beauv.; Pooideae; 1 especie.

1. B. mexicanum (Roem. \& Schult.) Link; (COX, CUI, HUA, IXT, TEP, TLA)

Briza L.; Pooideae; 1 especie.

1. B. minor L.; (IXT) 
Apéndice. Continuación.

Bromus L.; Pooideae; 5 especies.

1. B. anomalus Rupr. ex E. Fourn.; (CEN, COX, HUA, IXT)

2. B. carinatus Hook. \& Arn.; (CEN, IXT, JUX, MIX, TEO, TEP, TUX)

3. B. dolichocarpus Wagnon; (CEN)

4. B. exaltatus Bernh.; (CEN, TEP)

5. B. porteri (J. M. Coult.) Nash; (IXT, MIX)

Calamagrostis Adans.; Pooideae; 4 especies.

1. C. erectifolia Hitchc.; (IXT)

2. C. intermedia (J. Presl) Steud.; (IXT)

3. C. orizabae Steud.; registrada por Espejo et al. (2000). No se especifica la localidad dentro del estado.

4. C. tolucensis (Kunth) Trin. ex Steud.; registrada por Espejo et al. (2000). No se especifica la localidad dentro del estado.

Cathestecum J. Presl; Chloridoideae; 3 especies, una representada por dos variedades.

1. C. brevifolium Swallen var. brevifolium; (CUI, JUC, TEH)

1. C. brevifolium Swallen var. hirsutum; (JUC, TEH)

2. C. prostratum J. Presl; (HUA)

3. C. varium Swallen; (CUI, TEH, TEO)

Cenchrus L.; Panicoideae; 8 especies.

1. C. brownii Roem. \& Schult.; (NOC, PUT, TUX)

2. C. ciliaris L.; (CUI, JUC, POC, TEH, TEO)

3. C. echinatus L.; (CEN, CUI, PUT, TEH, TEO, TUX)

4. C. incertus M. A. Curtis; (CUI, TEH)

5. C. multiflorus J. Presl; (JUC, JUQ, TEH, TUX)

6. C. myosuroides Kunth; (HUA, IXT, NOC)

7. C. pilosus Kunth; (CUI, JUC, TEH)

8. C. tribuloides L.; (HUA)

Chaetium Nees; Panicoideae; 1 especie.

1. C. bromoides (J. Presl) Benth.; (JUX)

Chascolytrum Desv.; Pooideae; 1 especie.

1. C. subaristatum (Lam.) Desv.; (IXT)

Chloris Sw.; Chloridoideae; 5 especies.

1. C. ciliata Sw.; (CUI)

2. C. gayana Kunth; (IXT, TUX) 
Apéndice. Continuación.

3. C. rufescens Lag.; (CEN, CUI)

4. C. submutica Kunth; (HUA, TEP)

5. C. virgata Sw.; (COX, CUI, IXT, TEH, TEO)

Chusquea Kunth; Bambusoideae; 9 especies, una representada por 2 subespecies.

1. C. aperta L. G. Clark; (IXT, TUX); endémica para Oaxaca.

2. C. bilimekii E. Fourn.; (IXT)

3. C. circinata Soderstr. \& C. E. Calderón; (JUC, TEH)

4. C. galeottiana Rupr. ex Munro; registrada por Espejo et al. (2000). No se especifica la localidad dentro del estado.

5. C. liebmannii E. Fourn.; (JUC, TEH)

6. C. mulleri Munro; (TEO)

7. C. perotensis L. G. Clark, G. Cortés \& Cházaro; registrada por Espejo et al. (2000). No se especifica la localidad dentro del estado.

8. C. pittieri Hack.; registrada por Espejo et al. (2000). No se especifica la localidad dentro del estado.

9. C. repens L. G. Clark \& Londoño subsp. oaxacensis L. G. Clark \& Londoño; (IXT); endémica para Oaxaca.

9. C. repens L. G. Clark \& Londoño subsp. repens; (MIX)

Cinna L.; Pooideae; 1 especie.

1. C. poiformis (Kunth) Scribn. \& Merr.; registrada por Espejo et al. (2000). No se especifica la localidad dentro del estado.

Coix L.; Panicoideae; 1 especie.

1. C. lacryma-jobi L.; (IXT)

Cottea Kunth; Chloridoideae; 1 especie.

1. C. pappophoroides Kunth; (CUI)

Cryptochloa Swallen; Bambusoideae; 1 especie.

1. C. strictiflora (E. Fourn.) Swallen; (JUC)

Ctenium Panzer; Chloridoideae; 2 especies.

1. C. planifolium (J. Presl) Kunth; registrada por Espejo et al. (2000). No se especifica la localidad dentro del estado.

2. C. plumosum (Hitchc.) Swallen; (JUQ)

Cyclostachya J. Reeder \& C. G. Reeder; Chloridoideae; 1 especie; endémica para México.

1. C. stolonifera (Scribn.) J. Reeder \& C. G. Reeder; (HUA) 
Apéndice. Continuación.

Cymbopogon Spreng.; Panicoideae; 2 especies.

1. C. citratus (DC.) Stapf; (JUC)

2. C. nardus (L.) Rendle; registrada por Espejo et al. (2000). No se especifica la localidad dentro del estado.

Cynodon Rich.; Chloridoideae; 3 especies.

1. C. dactylon (L.) Pers.; (CEN, CUI, IXT, OCO, SOL, TEH, TLA, TUX)

2. C. nlemfuensis Vanderyst; (JUC)

3. C. plectostachyus (K. Schum.) Pilg.; (IXT, TEH, TUX)

Dactylis L.; Pooideae; 1 especie.

1. D. glomerata L.; (CEN)

Dactyloctenium Willd.; Chloridoideae; 1 especie.

1. D. aegyptium (L.) Willd.; (JAM, JUX, TEH, TUX)

Dichanthelium (Hitchc. \& Chase) Gould; Panicoideae; 9 especies.

1. D. acuminatum (Sw.) Gould \& C. A. Clark var. acuminatum; (IXT, SOL)

2. D. commutatum (Schult.) Gould; (JUX, IXT)

3. D. dichotomum (L.) Gould; (IXT, TEH)

4. D. laxiflorum (Lam.) Gould; (CHO, MIX, TEO, VAL)

5. D. pedicellatum (Vasey) Gould; (MIX)

6. D. sphaerocarpon (Elliott) Gould var. sphaerocarpon; (IXT, TEH)

7. D. strigosum (Muhl. ex Elliott) Freckmann; registrada por Espejo et al. (2000). No se especifica la localidad dentro del estado.

8. D. umbonulatum (Swallen) Davidse; (TLA)

9. D. viscidellum Scribn.; (IXT)

Dichanthium Willemet; Panicoideae; 1 especie.

1. D. annulatum (Forssk.) Stapf; (CEN, TEH, TUX)

Digitaria Haller; Panicoideae; 18 especies.

1. D. argillacea (A. Hitchc. \& Chase) Fernald; (CEN, ETL)

2. D. badia (Scribn. \& Merr.) Fernald; (POC, TUX)

3. D. bicornis (Lam.) Roem. \& Schult.; (CUI, IXT, JUC, JUX, POC, TEH, TUX)

4. D. cayoënsis Swallen; (JUC)

5. D. ciliaris (Retz.) Koeler; (CHO, CUI, JUC, JUX, POC, TLA, TUX)

6. D. curtigluma Hitchc.; (CEN, JUX, TUX)

7. D. filiformis (L.) Koeler; (JUX)

8. D. hitchcockii (Chase) Stuck.; registrada por Espejo et al. (2000). No se especifica la localidad dentro del estado. 
Apéndice. Continuación.

9. D. horizontalis Willd.; (TUX)

10. D. insularis (L.) Fedde; (CEN, JUC, TLA, TEH, TUX)

11. D. leucites (Trin.) Henr.; (TUX)

12. D. nuda Schumach.; registrada por Espejo et al. (2000). No se especifica la localidad dentro del estado.

13. D. panicea (Sw.) Urb.; registrada por Espejo et al. (2000). No se especifica la localidad dentro del estado.

14. D. pentzii Stent.; registrada por Davidse et al. (1994) y Espejo et al. (2000). No se especifica la localidad dentro del estado.

15. D. sanguinalis (L.) Scop.; (TUX)

16. D. setigera Roth ex Roem. \& Schult. var. setigera; (TUX)

17. D. ternata (A. Rich.) Stapf; (TUX)

18. D. villosa (Walter) Pers.; (JUC)

Distichlis Raf.; Chloridoideae; 1 especie.

1. D. spicata (L.) Greene; (TEH, TEO, TEP)

Echinochloa P. Beauv.; Panicoideae; 5 especies.

1. E. colona (L.) Link; (CEN, JUC, JUX, OCO, POC, TEH, TEO, TUX)

2. E. crus-pavonis (Kunth) Schult.; (CEN, POC, TEO)

3. E. oplismenoides (E. Fourn.) A. Hitchc.; (TEP)

4. E. polystachya (Kunth) A. Hitchc.; (JUC, TUX)

5. E. pyramidalis (Lam.) A. Hitchc. \& Chase; (JUC, THE, TUX)

Eleusine Gaertn.; Chloridoideae; 1 especie.

1. E. indica (L.) Gaertn.; (CHO, JUC, JUX, PUT, TEO)

Elionurus Kunth ex Willd.; Panicoideae, 1 especie, representada por una variedad

1. E. tripsacoides Kunth ex Willd. var. tripsacoides; (TEP)

Elymus L.; Pooideae; 1 especie.

1. E. xpseudorepens (Scribn. \& J. G. Sm. (pro sp.)) Barkworth \& D. R. Dewey; (TLA)

Enneapogon Desv. ex P. Beauv.; Chloridoideae; 1 especie.

1. E. desvauxii P. Beauv.; (CEN, CUI, HUA)

Enteropogon Nees; Chloridoideae; 1 especie.

1. E. chlorideus (J. Presl) Clayton; (CUI) 
Apéndice. Continuación.

Eragrostis Wolf; Chloridoideae; 28 especies, de las cuales tres están representadas por variedades y una por subespecie.

1. E. acutiflora (Kunth) Nees

2. E. amabilis (L.) Wight \& Arn. ex Nees ; (POC)

3. E. atrovirens (Desf.) Trin. ex Steud.; (TEP)

4. E. barrelieri Daveau; (CUI, TEP)

5. E. cilianensis (All.) Vignolo ex Janch.; (CUI, TEO, TEH)

6. E. ciliaris (L.) R. Br. var. ciliaris; (JAM, POC, TEH, TUX)

7. E. elliottii Watson; registrada por Espejo et al. (2000). No se especifica la localidad dentro del estado.

8. E. glomerata (Walter) L. H. Dewey; (TEH)

9. E. hirsuta (Mix.) Nees; (CUI, TEO, TEH)

10. E. hirta E. Fourn. var. longiramea (Swallen) Withers; (CUI, TEO)

11. E. hondurensis R. W. Pohl; (JUC)

12. E. hypnoides (Lam.) Britton, Sterns \& Poggenb.; (JUC, TEH)

13. E. intermedia Hitchc.; (COX, IXT, SOL, TEH, TEO)

14. E. japonica (Thunb.) Trin.; (TEH)

15. E. lugens Nees; (IXT, POC, PUT)

16. E. maypurensis (Kunth) Steud.; (JAM, PUT, TEH, TUX)

17. E. mexicana (Hornem.) Link subsp. mexicana; (CEN, COX, CUI, HUA, IXT, TEP, ZAA)

18. E. obtusiflora (E. Fourn.) Scribn.; (CEN)

19. E. palmeri S. Watson; (CEN, POC)

20. E. pectinacea var. miserrima (E. Fourn.) Reeder (IXT)

21. E. pilosa (L.) P. Beauv.; (CEN, TEH, TUX)

22. E. polytricha Nees; registrada por Espejo et al. (2000). No se especifica la localidad dentro del estado.

23. E. prolifera (Sw.) Steud.; (JUC, TEH)

24. E. secundiflora J. Presl; (TEH, TUX)

25. E. silveana Swallen; (TEO)

26. E. swallenii A. Hitchc.; (COX, CUI, NOC)

27. E. tenuifolia (A. Rich) Hochst. ex Steud.; (TEH)

28. E. viscosa (Retz.) Trin.; (COX)

Eriochloa Kunth; Panicoideae; 5 especies, cuatro de las cuales están representadas por variedades.

1. E. acuminata (J. Presl) Kunth var. minor (Vasey) R. B. Shaw; (CEN)

2. E. aristata Vasey var. aristata; (JUC)

2. E. aristata Vasey var. boxiana (Hitchc. ex Swallen) R. B. Shaw; registrada por Espejo et al. (2000). No se especifica la localidad dentro del estado. 
Apéndice. Continuación.

3. E. contracta Hitchc.; (JAM)

4. E. nelsonii Scribn. \& G. Sm. var. nelsonii; (TEH, YAU)

5. E. punctata (L.) Desv. ex Ham.; (CUI)

Eriochrysis P. Beauv.; Panicoideae; 1 especie.

1. E. cayennensis P. Beauv.; registrada por Espejo et al. (2000). No se especifica la localidad dentro del estado.

Erioneuron Nash.; Chloridoideae; 2 especies.

1. E. avenaceum (Kunth) Tateoka; (COX, HUA, NOC, TEP)

2. E. pilosum (Buckey) Nash; (POC)

Euclasta Franch.; Panicoideae; 1 especie.

1. E. condylotricha (Hochst. ex Steud.) Stapf; registrada por Espejo et al. (2000). No se especifica la localidad dentro del estado.

Festuca L.; Pooideae; 5 especies.

1. F. amplissima Rupr.; (IXT, JUX)

2. F. breviglumis Swallen; (JUX, POC)

3. F. callosa (Piper) St.-Yves; (CUI, TEO)

4. F. lugens (E. Fourn.) A. Hitchc. ex Hern.-Xol.; (CUI, MIX, POC)

5. F. willdenoviana Schult. \& Schult.; registrada por Espejo et al. (2000). No se especifica la localidad dentro del estado.

Glyceria R. Br.; Pooideae; 1 especie, representada por una variedad.

1. G. striata (Lam.) Hitchc. var. mexicana Kelso; registrada por Espejo et al. (2000). No se especifica la localidad dentro del estado.

Gouinia E. Fourn. ex Benth. \& Hook. f.; Chloridoideae; 2 especies, representadas por variedades.

1. G. latifolia (Griseb.) Vasey var. guatemalensis (Hack.) J. J. Ortiz; (VAL)

2. G. virgata (J. Presl) Scribn. var. virgata; (VAL)

Guadua Kunth; Bambusoideae; 5 especies, una de las cuales está representada por 1 variedad.

1. G. aculeata Rupr. ex E. Fourn.; (JUC, TUX)

2. G. amplexifolia J. Presl; (TUX)

3. G. angustifolia Kunth var. angustifolia; (JUC, TUX)

4. G. longifolia (E. Fourn.) R. W. Pohl; (POC)

5. G. paniculata Munro; (JUC, POC, TUX) 
Apéndice. Continuación.

Gynerium Willd. ex P. Beauv.; Arundinoideae; 1 especie.

1. G. sagittatum (Aubl.) P. Beauv.; (TEH)

Heteropogon Pers.; Panicoideae; 2 especies.

1. H. contortus (L.) P. Beauv.; (CEN, CUI, ETL, HUA, JUC, JUX, OCO, SOL, TEH, TLX)

2. H. melanocarpus (Elliott) Benth.; (CEN, ETL)

Hierochloë R. Br.; Pooideae; 1 especie.

1. H. mexicana (Rupr. ex E. Fourn.) Benth. ex A. Hitchc.; (CEN, IXT)

Hilaria Kunth; Chloridoideae; 3 especies, una representada por 1 variedad.

1. H. belangeri (Steud.) Nash var. belangeri; (HUA)

2. H. cenchroides Kunth; (CEN, COX, TEP, TLA)

3. H. ciliata (Scribn.) Nash; (COX, THE)

Homolepis Chase; Panicoideae; 2 especies.

1. H. aturensis (Kunth) Chase; (JUC, TUX)

2. H. glutinosa (Sw.) Zuloaga \& Soderstr.; (IXT)

Hordeum L.; Pooideae; 1 especie.

1. H. vulgare L.; (TEP)

Hymenachne P. Beauv.; Panicoideae; 2 especies.

1. H. amplexicaulis (Rudge) Nees; (JAM, TUX)

2. H. donacifolia (Raddi) Chase; (TUX)

Hyparrhenia Anderss.; Panicoideae; 3 especies.

1. H. bracteata (Humb. \& Bonpl. ex Willd.) Stapf; (TEH,TUX)

2. H. hirta (L.) Stapf; (JUC)

3. H. rufa (Nees) Stapf; (TLX, TUX)

Hypogynium Nees; Panicoideae; 1 especie.

1. H. virgatum (Desv.) Dandy; registrada por Espejo et al. (2000). No se especifica la localidad dentro del estado.

Ichnanthus P. Beauv.; Panicoideae; 3 especies.

1. I. nemorosus (Sw.) Doell; (IXT)

2. I. pallens (Sw.) Munro ex Benth.; (CHO, JUC, PUT)

3. I. tenuis (J. Presl) Hitchc.; (TUX) 
Apéndice. Continuación.

Imperata Cirillo; Panicoideae; 1 especie.

1. I. brasiliensis Trin.; (CHO, TUX)

Isachne R. Br.; Panicoideae; 1 especie.

1. I. arundinacea (Sw.) Griseb.; (CHO, IXT, JUC, MIX, TEH, TUX, VAL)

Ischaemum L.; Panicoideae; 1 especie.

1. I. latifolium (Spreng.) Kunth; registrada por Espejo et al. (2000). No se especifica la localidad dentro del estado.

Ixophorus Schltdl.; Panicoideae; 1 especie.

1. I. unisetus (J. Presl) Schltdl.; (JAM)

Jarava Ruiz \& Pav.; Pooideae; 1 especie.

1. J. ichu Ruiz \& Pav.; (COX, HUA, JUX, TEP, TLA, ZAA)

Jouvea E. Fourn.; Chloridoideae; 2 especies.

1. J. pilosa (J. Presl) Scribn.; (TEH)

2. J. straminea E. Fourn.; (CUI, TEH)

Koeleria Pers.; Pooideae; 1 especie.

1. K. pyramidata (Lam.) P. Beauv.; (CEN)

Lasiacis (Griseb.) A. Hitchc.; Panicoideae; 10 especies, tres representadas por variedades.

1. L. divaricata (L.) A. Hitchc. var. divaricata; (COX, JUC, MIA, MIX, TEH, TUX)

1. L. divaricata (L.) A. Hitchc. var. leptostachya (A. Hitchc.) Davidse; (COX, JUC)

2. L. grisebachii (Nash) A. Hitchc. var. grisebachii; (TUX)

3. L. nigra Davidse; (COX, CUI, ETL, HUA, IXT, JUC, MIA, MIX, TEH, TEO, TLA, TLX)

4. L. oaxacacensis (Steud.) A. Hitchc. var. oaxacacensis; (JUC, TUX)

5. L. procerrima (Hack.) A. Hitchc.; (CHO, JAM, JUC, JUQ, MIA, POC, PUT, TEH, TUX)

6. L. rhizophora (E. Fourn.) A. Hitchc.; (JUQ, POC, TEH)

7. L. ruscifolia (Kunth) A. Hitchc. var. ruscifolia; (JAM, JUC, JUQ, POC, TEH, TUX)

8. L. scabrior A. Hitchc.; (JUC, JUQ, TUX)

9. L. sloanei (Griseb.) A. Hitchc.; (TUX)

10. L. sorghoidea (Desv.) A. Hitchc. \& Chase; (JUC, TEH, TUX, YAU)

Leersia Sw.; Bambusoideae; 2 especies.

1. L. hexandra Sw.; (TEP)

2. L. ligularis Trin.; (JUC) 
Apéndice. Continuación.

Leptochloa P. Beauv.; Chloridoideae; 6 especies, dos representadas por subespecies.

1. L. dubia (Kunth) Nees; (COX, CUI, TEP)

2. L. fusca (L.) Kunth ssp. uninervia (J. Presl) N. Snow; (TEH, TEO)

3. L. panicea (Retz.) Ohwi ssp. mucronata (Michx.) Nowack; (CUI, TEH)

4. L. panicoides (J. Presl) A. Hitchc.; registrada por Espejo et al. (2000). No se especifica la localidad dentro del estado.

5. L. uninervia (J. Presl) A. Hitchc.; (TEH)

6. L. virgata (L.) P. Beauv.; (JUC, TUX)

Lithachne P. Beauv.; Bambusoideae; 1 especie.

1. L. pauciflora (Sw.) P. Beauv.; (POC)

Lolium L.; Pooideae; 4 especies.

1. L. arundinaceum (Schreb.) Darbysh.; (IXT, TEO)

2. L. multiflorum Lam.; (IXT)

3. L. perenne L.; registrada por Espejo et al. (2000). No se especifica la localidad dentro del estado.

4. L. temulentum L.; registrada por Espejo et al. (2000). No se especifica la localidad dentro del estado.

Luziola A. L. Juss.; Bambusoideae; 1 especie.

1. L. peruviana J. F. Gmel; (POC, PUT)

Lycurus Kunth; Chloridoideae; 2 especies.

1. L. phalaroides Kunth; (NOX, PUT, SOL)

2. L. phleoides Kunth.; (CEN, IXT, NOX, TEP)

Melica L.; Pooideae; 1 especie.

1. M.porteri Scribn.; (TEP)

Melinis P. Beauv.; Panicoideae; 2 especies.

1. M. minutiflora P. Beauv.; (JUQ, TEO)

2. M. repens (Willd.) Zizka; (CEN, CUI, IXT, JUC, TEH, YAU, TEP, TLA, TLX, TUX, ZIM)

Mesosetum Steud.; Panicoideae; 2 especies.

1. M.filifolium C. E. Hubb.; (TUX); endémica para Oaxaca.

2. M.pittieri A. Hitchc.; (JUC)

Metcalfia Conert; Pooideae; 1 especie, endémica para México.

1. M. mexicana (Scribn.) Conert; (HUA, NOC) 
Apéndice. Continuación.

Microchloa R. Br.; Chloridoideae; 2 especies.

1. M.indica (L.) P. Beauv.; (CEN, CUI)

2. M. kunthii Desv.; (NOC, TEO)

Mnesithea Kunth; Panicoideae; 1 especie.

1. M.granularis (L.) de Koning \& Sosef; (JUC, TEH, TUX, VAL)

Monanthochloë Engelm.; Chloridoideae; 1 especie.

1. M. littoralis Engelm.; (TEH)

Muhlenbergia Schreb.; Chloridoideae; 33 especies.

1. M. capillaris (Lam.) Trin.; (CEN, JUX, TLA)

2. M. ciliata (Kunth) Trin.; (JUX, TEH, TLA)

3. M. depauperata Scribn.; (COX)

4. M. distans Swallen; (HUA, JUC, JUX, TEP, TUX)

5. M. distichophylla (J. Presl) Kunth; (MIX, HUA, TEP)

6. M. dubia E. Fourn.; (CUI)

7. M.dumosa Scribn. ex Vasey (TEH)

8. M. emersleyi Vasey; (ETL, HUA, IXT, TEO)

9. M. fragilis Swallen; (TEH)

10. M. gigantea (E. Fourn.) A. Hitchc.; (MIX, POC)

11. M. implicata (Kunth) Kunth; (CEN, HUA, TEP)

12. M. macroura (Kunth) Hitchc.; (POC)

13. M. microsperma (DC.) Kunth; (JUX, HUA, TLX)

14. M.minutissima (Steud.) Swallen; (IXT)

15. M. montana (Nutt.) A. Hitchc.; (COX, CUI, JUX, NOC)

16. M. mucronata (Kunth) Trin.; (CEN)

17. M. nigra A. Hitchc.; (CEN, HUA)

18. M.pectinata Goodding; (TEH)

19. M.peruviana (P. Beauv.) Steud.; (COX)

20. M.pubescens (Kunth) Hitchc.; (TEH)

21. M.quadridentata (Kunth) Kunth; (IXT)

22. M. ramulosa (Kunth) Swallen; (VAL)

23. M.repens (J. Presl) Hitchc.; (TEH)

24. M. rigida (Kunth) Kunth; (IXT, TEO, TEP)

25. M. robusta (E. Fourn.) A. Hitchc.; (CUI, JUC, MIA, SIL, TEH, TEO, TEP)

26. M. spiciformis Trin.; (CEN)

27. M.stricta (J. Presl) Kunth; registrada por Espejo et al. (2000). No se especifica la localidad dentro del estado.

28. M. subaristata Swallen; registrada por Espejo et al. (2000). No se especifica la localidad dentro del estado. 
Apéndice. Continuación.

29. M.tenella (Kunth) Trin.; (JAM, JUC, POC, TEH)

30. M. tenuifolia (Kunth) Kunth; (CEN, HUA, TEP, PUT)

31. M. vaginata Swallen; (JUX, TEP)

32. M. versicolor Swallen; (POC, VAL)

33. M. virletii (E. Fourn.) Soderstr.; (IXT)

Nassella Desv.; Pooideae; 5 especies.

1. N. lepida (Hitchc.) Barkworth; (HUA)

2. N. linearifolia (E. Fourn.) R. W. Pohl; (COX, HUA)

3. N. mucronata (Kunth) R. W. Pohl; (COX, TEP)

4. N. mexicana (Hitchc.) R. W. Pohl; (COX)

5. N. tenuissima (Trin.) Barkworth; registrada por Espejo et al. (2000). No se especifica la localidad dentro del estado.

Olmeca Soderstr.; Bambusoideae; 1 especie; endémica para México.

1. O. reflexa Swallen; (IXT, JUC)

Olyra L.; Bambusoideae; 2 especies.

1. O. glaberrima Raddi; (JUC, POC, TUX)

2. O. latifolia L.; (CHO, JAM, JUC, POC, TUX)

Oplismenus P. Beauv.; Panicoideae; 3 especies, una está representada por 2 variedades y otra por 1 subespecie.

1. O. burmannii (Retz.) P. Beauv. var. burmannii; (JUC, ETL, POC, PUT, TEH)

1. O. burmannii (Retz.) P. Beauv. var. nudicaulis (Vasey) McVaugh; (CEN, CUI, JAM, JUC, JUX, POC, TEH)

2. O. compositus (L.) P. Beauv.; (CEN, ETL, JAM, JUC, MIA, POC, PUT, TEO, TEP)

3. O. hirtellus (L.) P. Beauv. subsp. hirtellus; (ETL, JUC, MIA, TEH, TUX)

Orthoclada P. Beauv.; Centothecoideae; 1 especie.

1. O. laxa (Rich.) P. Beauv.; (CHO, JUC)

Oryza L.; Bambusoideae; 3 especies.

1. O. latifolia Desv. (JUC, JUQ)

2. O. rufipogon Griff.; (TEH)

3. O. sativa L.; (CEN, ETL, TEH)

Otatea (McClure \& Smith) C. E. Calderón \& Soderstr.; Bambusoideae; 2 especies, una de las cuales está representada por 1 subespecie.

1. O. acuminata (Munro) C. E. Calderón \& Soderstr. subsp. acuminata; (COX, CUI, NOC, SIL, TEH, TEP, TUX)

2. O. fimbriata Soderstr.; (ETL) 
Apéndice. Continuación.

Panicum L.; Panicoideae; 26 especies, tres de las cuales están representadas por variedades.

1. P. alatum Zuloaga \& Morrone var. minus (Andersson) Zuloaga \& Morrone; (SOL, TUX)

2. P. arundinariae Trin. ex E. Fourn.; registrada por Espejo et al. (2000). No se especifica la localidad dentro del estado.

3. P. biglandulare Scribn. \& J. G. Sm.; (JUX, MIA, POC, PUT)

4. P. bulbosum Kunth; (CEN, IXT, MIA, MIX, TEH)

5. P. cayennense Lam.; (TUX)

6. P. crateriferum Sohns; (MIX)

7. P. decolorans Kunth; (CEN, IXT)

8. P. hallii Vasey var. hallii; (HUA)

9. P. hirticaule J. Presl var. hirticaule; (CUI, JUC, TEH, TUX)

10. P. hirtum Lam.; (POC)

11. P. hispidifolium Swallen; registrada por Espejo et al. (2000). No se especifica la localidad dentro del estado.

12. P. mertensii Roth; (MIA)

13. P. miliaceum L.; (CUI)

14. P. parcum Hitchc. \& Chase; (CUI)

15. P. parviglume Hack.; (MIA)

16. P. pilosum Sw.; (JUC, PUT, TUX)

17. P. polygonatum Schrad.; (JUC)

18. P. pulchellum Raddi; (CHO, IXT, POC, JUC, TUX)

19. P. stoloniferum Poir.; (TUX)

20. P. stramineum Hitchc. \& Chase; (JUC)

21. P. sublaeve Swallen; (CEN)

22. P. trichanthum Nees; (TUX)

23. P. trichidiachne Döll; (POC, PUT, IXT)

24. P. trichoides Sw.; (CUI, JAM, JUC, POC, PUT, TEH, TUX, YAU)

25. P. tuerckheimii Hack.; (TUX)

26. P. virgatum L.; (POC, SOL, TEH, TLA, TUX)

Pappophorum Schreb.; Chloridoideae; 1 especie.

1. P. pappiferum (Lam.) Kuntze; (CUI, NOC)

Paspalidium Stapf; Panicoideae; 1 especie

1. P. geminatum (Forssk.) Stapf; (TEH)

Paspalum L; Panicoideae; 41 especies.

1. P. affine Steud.; registrada por Davidse et al. (1994) y Espejo et al. (2000). No se especifica la localidad dentro del estado. 
Apéndice. Continuación.

2. P. blodgettii Chapm.; (IXT, JAM, TLA, TUX)

3. P. botterii (E. Fourn.) Chase; (CUI, SOL)

4. P. clavuliferum C. Wright ; registrada por Espejo et al. (2000). No se especifica la localidad dentro del estado.

5. P. conjugatum Bergius; (CEN, CUI, JUC, JUX, PUT, TEO, TEP, TUX)

6. P. conspersum Schrad.; registrada por Espejo et al. (2000). No se especifica la localidad dentro del estado.

7. P. convexum Humb. \& Bonpl. ex Flüggé; (JUC, JUX, MIA, POC)

8. P. corcovadense Raddi; registrada por Davidse et al. (1994) y Espejo et al. (2000): No se especifica la localidad dentro del estado.

9. P. costaricense Mez.; registrada por Davidse et al. (1994). No se especifica la localidad dentro del estado.

10. P. cymbiforme E. Fourn.; (CEN, TLA)

11. P. denticulatum Trin.; (CUI, HUA, NOC, TEH, TLA)

12. P. distichum L.; (CEN, CUI, HUA, IXT)

13. P. erianthum Nees ex Trin.; (TEO)

14. P. fasciculatum Willd. ex Flüggé; (HUA, TEO)

15. P. hartwegianum E. Fourn.; registrada por Espejo et al. (2000). No se especifica la localidad dentro del estado.

16. P. hintonii Chase; (JUX)

17. P. humboldtianum Flüggé; (IXT, MIX, NOC, TEH, TLX)

18. P. jaliscanum Chase; (IXT)

19. P. langei (E. Fourn.) Nash; (TEO, TUX)

20. P. lentiginosum J. Presl; (TEH)

21. P. ligulare Nees; (CEN, TEH). Esta especie sólo se conoce de Oaxaca.

22. P. longiscupe Nash; (JUQ)

23. P. melanospermum Desv. ex Poir.; (JAM)

24. P. minus E. Fourn.; registrada por Espejo et al. (2000). No se especifica la localidad dentro del estado.

25. P. multicaule Poir.; (POC)

26. P. mutabile Chase; registrada por Espejo et al. (2000). No se especifica la localidad dentro del estado.

27. P. notatum Flüggé; (CEN, CUI, JUC, NOC, TLA)

28. P. orbiculatum Poir.; (TUX)

29. P. paniculatum L.; (POC, TEH, TUX)

30. $P$. pectinatum Nees ex Trin.; (CHO, JUC, JUC)

31. P. plicatulum Michx.; (CHO, JUC, PUT, TUX, ZIM)

32. P. prostratum Scribn. \& Merr.; (MIA, PUT)

33. P. pubiflorum Rupr. ex E. Fourn.; (CEN, TLA)

34. P. pulchellum Kunth; (PUT)

35. P. squamulatum E. Fourn.; (TLA) 
Apéndice. Continuación.

36. P. stellatum Humb. \& Bonpl. ex Flüggé; (JUQ)

37. P. tinctum Chase; registrada por Espejo et al. (2000). No se especifica la localidad dentro del estado.

38. P. trachycoleon Steud.; (TEH)

39. P. unispicatum (Scribn. \& Merr.) Nash; (CEN, ETL)

40. P. variabile (E. Fourn.) Nash; (TEH)

41. P. virgatum L.; (CEN, CHO, JUC, PUT, TUX)

Pennisetum Rich.; Panicoideae; 8 especies.

1. P. bambusiforme (E. Fourn.) Hemsl. ex Jack.; (POC, SOL)

2. P. crinitum (Kunth) Spreng.; (JUX, SOL)

3. P. distachyum (E. Fourn.) Rupr. ex Chase; (CUI, HUA)

4. P. durum Beal; (TUX)

5. P. nervosum (Nees) Trin.; (TUX)

6. P. polystachion (L.) Schult.; (JAM, JUC, PUT, TEH, TUX, VAL, YAU)

7. P. prolificum Chase; (TEO)

8. P. purpureum Schumach.; (IXT, JUX, TEH, TUX)

Pereilema J. Presl; Chloridoideae; 2 especies.

1. P. ciliatum E. Fourn.; (JUX, PUT)

2. P. crinitum J. Presl; (CUI)

Peyritschia E. Fourn.; Pooideae; 2 especies

1. P. koelerioides (Peyr.) E. Fourn.; (CEN)

2. P. deyeuxioides (Kunth) Finot; (CUI, IXT, TLA, TEP)

Phalaris L.; Pooideae; 1 especie.

1. P. canariensis L.; (NOC, TEP)

Pharus P. Br.; Bambusoideae; 2 especies.

1. P. latifolius L.; (JUC)

2. P. parvifolius Nash; (JUC)

Phyllostachys Siebold \& Zucc.; Bambusoideae; 1 especie.

1. P. aurea Rivière \& C. Rivière; registrada por Espejo et al. (2000). No se especifica la localidad dentro del estado.

Piptochaetium J. Presl; Pooideae; 3 especies.

1. P. angustifolium (Hitchc.) Valencia \& Costas; (COX)

2. P. fimbriatum (Kunth) A. Hitchc.; (COX, NOC, TEP)

3. P. virescens (Kunth) Parodi; (CEN, COX, CUI, JUX) 
Apéndice. Continuación.

Poa L.; Pooideae; 5 especies.

1. P. annua L.; (CEN, IXT, TEP, TUX)

2. P. bigelovii Vasey \& Scribn.; (IXT)

3. P. ruprechtii Peyr; (TEP)

4. P. scaberula Hook. f.; (IXT)

5. P. seleri Pilg.; (IXT)

Polypogon Desf.; Pooideae; 3 especies.

1. P. elongatus Kunth; (COX, HUA)

2. P. monspeliensis (L.) Desf.; (CUI, IXT, TEP)

3. P. viridis (Gouan) Breistr.; (CUI, HUA, IXT, JUX, TEP)

Pseudechinolaena Stapf; Panicoideae; 1 especie.

1. P. polystachya (Kunth) Stapf; (IXT, MIA, POC, TEH)

Reimarochloa A. Hitchc.; Panicoideae; 1 especie.

1. R. acuta (Flüggé) A. Hitchc.; (TEH)

Rhipidocladum McClure; Bambusoideae; 1 especie.

1. $R$. racemiflorum (Steud.) McClure; (TUX)

Rottboellia L.; Panicoideae; 1 especie.

1. R. cochinchinensis (Lour.) W. Clayton; (TUX)

Saccharum L.; Panicoideae; 1 especie.

1. S. officinarum L.; (IXT, ZIM)

Schedonorus P. Beauv.; Pooideae; 1 especie

1. S. arundinaceus (Schreb.) Dumort.; (IXT, TEO)

Schizachyrium Nees; Panicoideae; 8 especies.

1. S. brevifolium (Sw.) Nees ex Büse; (TLX, JUC)

2. S. cirratum (Hack.) Wooton \& Standl.; (TLA, IXT)

3. S. condensatum (Kunth) Nees; (JUC, POC, TEH, TUX)

4. S. microstachyum (Desv. ex Ham.) Roseng., B. R. Arrill. \& Izag.; (TUX, JUC)

5. S. salzmannii (Trin. ex Steud.) Nash.; registrada por Manrique (1994) y Espejo et al. (2000). No especifica la localidad dentro del estado.

6. S. sanguineum (Retz.) Alston; (CUI, HUA, IXT, JUX, JUC, NOC, POC, PUT, SIL, TUX)

7. S. semitectum (Swallen) J. Reeder; (TEH)

8. S. tenerum Nees; (HUA, JUX, JUC) 
Apéndice. Continuación.

Setaria $\boldsymbol{P}$. Beauv.; Panicoideae; 14 especies, una está representada por 1 variedad.

1. S. adhaerens (Forssk.) Chiov.; (CEN, TLA)

2. S. grisebachii E. Fourn.; (CEN, COX, CUI, HUA, JUC, NOC, TEO, TLA)

3. S. latifolia (Scribn.) Herrm.; registrada por Rominger (1962) y Espejo et al. (2000). No se especifica la localidad dentro del estado.

4. S. leucopila (Scribn. \& Merr.) K. Schum.; (CUI)

5. S. liebmannii E. Fourn.; (JUC, POC, TEH, TLA)

6. S. longipila E. Fourn.; registrada por Espejo et al. (2000). No se especifica la localidad dentro del estado.

7. S. macrostachya Kunth; (CUI, NOC, TEO)

8. S. palmifolia (J. König) Stapf; (TEH, TUX)

9. S. parviflora (Poir.) Kerguélen; (CEN, COX, CUI, HUA, IXT, JUC, MIX, PUT, SIL, TEH, TEP, TLA, TLX, TUX)

10. S. poiretiana (Schult.) Kunth; (IXT, TUX)

11. S. scandens Schrad.; (VAL)

12. S. sulcata Raddi; (IXT, TUX)

13. S. tenax (Rich.) Desv. var. tenax; (JUC, TUX)

14. S. vulpiseta (Lam.) Roem. \& Schult.; (JUC, TUX)

Setariopsis Scribn.; Panicoideae; 2 especies.

1. S. auriculata (E. Fourn.) Scribn.; (CUI, TEO)

2. S. latiglumis (Vasey) Scribn.; (CUI)

Sorghastrum Nash; Panicoideae; 3 especies, una representada por 1 variedad.

1. S. brunneum Swallen; (ETL, IXT)

2. S. incompletum (J. Presl) Nash var. incompletum; (VAL)

3. S. nutans (L.) Nash; (CHO, HUA, MIA)

Sorghum Moench; Panicoideae; 3 especies.

1. S. bicolor (L.) Moench; (CEN, CHO, ETL, TUX)

2. S. halepense (L.) Pers.; (CUI, JUC, TEH)

3. S. trichocladum (Hack.) Kuntze; (HUA, IXT)

Sphenopholis Scribn.; Pooideae; 1 especie.

1. S. obtusata (Michx.) Scribn.; registrada por Espejo et al. (2000). No se especifica la localidad dentro del estado.

Sporobolus R. Br.; Chloridoideae; 9 especies.

1. S. airoides (Torr.) Torr.; (TEO)

2. S. atrovirens (Kunth) Kunth; (NOC, TEH, TLA)

3. S. cryptandrus (Torr.) A. Gray; (TEH) 
Apéndice. Continuación.

4. S. indicus (L.) R. Br.; (ETL, IXT, JUX, NOC, PUT, TEH, TEO, TEP, TLA, TUX)

5. S. jacquemontii Kunth; (JUC, PUT, TEH, TUX)

6. S. purpurascens (Sw.) Ham.; (TEP, TLA)

7. S. pyramidatus (Lam.) Hitchc.; (CUI, ETL, TEH, TEO)

8. S. splendens Swallen; (JUC)

9. S. virginicus (L.) Kunth; (TEH)

Steinchisma Raf.; Panicoideae; 1 especie

1. S. laxa (Sw.) Zuloaga; (JUC, JUX, TEH)

Streptochaeta Schrad.; Bambusoideae; 1 especie.

1. S. spicata Schrad. ex Nees subsp. spicata; (JUC, TUX)

Thrasya Kunth; Panicoideae; 1 especie.

1. T. campylostachya (Hack.) Chase; (JUC, TUX)

Trachypogon Nees; Panicoideae; 2 especies.

1. T. spicatus (L.f.) Kuntze ; (ETL, HUA, IXT, JUC, JUX, MIX, TEH, TLA, YAU)

2. T. vestitus Andersson; (HUA)

Tragus Haller; Chloridoideae; 1 especie.

1. T. berteronianus Schult.; (CEN, CUI, TEH, TEP)

Trichloris E. Fourn. ex Benth.; Chloridoideae; 1 especie.

1. T. pluriflora E. Fourn.; (CUI, TEO)

Tridens Roem. \& Schult.; Chloridoideae; 1 especie.

1. T. eragrostoides (Vasey \& Scribn.) Nash; registrada por Espejo et al. (2000). No se especifica la localidad dentro del estado.

Triniochloa A. Hitchc.; Pooideae; 2 especies.

1. T. gracilis Gomez-Sánchez \& M. González; registrada por Espejo et al. (2000). No se especifica la localidad dentro del estado.

2. T. stipoides (Kunth) Hitchc.; (CEN, JUX, SOL)

Tripsacum L.; Panicoideae; 5 especies, una representada por 1 variedad.

1. T. dactyloides (L.) L. var. mexicanum de Wet \& Harlan; (CEN, ETL, CUI, TEH)

2. T. latifolium A. Hitchc.; (TUX)

3. T. laxum Nash; (JUC, TUX)

4. T. maizar Hern.-Xol. \& Randolph; (PUT)

5. T. zopilotense Hern.-Xol. \& Randolph; (CEN, CUI, IXT, TEH, TEP) 
Apéndice. Continuación.

Trisetum Pers.; Pooideae; 3 especies.

1. T. irazuense (Kuntze) A. Hitchc.; (IXT, TEP)

2. T. pringlei (Scribn.) A. Hitchc.; (IXT, TLA)

3. T. viride (Kunth) Kunth; (CEN)

Tristachya Nees; Panicoideae; 1 especie.

1. T. avenacea (J. Presl) Scribn. \& Merr.; (HUA, PUT)

Triticum L.; Pooideae; 1 especie.

1. T. aestivum L.; (NOC, TEP)

Uniola L.; Chloridoideae; 1 especie.

1. U. pittieri Hack.; (JUQ, TEH)

Urochloa P. Beauv.; Panicoideae; 12 especies.

1. U. arizonica (Scribn. \& Merr.) Morrone \& Zuloaga; (CUI, TEH)

2. U. arrecta (Hack. ex T. Durand \& Schinz) Morrone \& Zuloaga; (JUC)

3. U. brizantha (Hochst. ex Rich) R. D. Webster; (TUX)

4. U. decumbens (Stapf) R. D. Webster; (JUC, TUX)

5. U. discifera (E. Fourn.) Morrone \& Zuloaga; (TEH)

6. U. fusca (Sw.) B. F. Hansen \& Wunderlin; (CEN, CUI, JUC, JUQ, POC, TEO, TEH, TUX)

7. U. maxima (Jacq.) R. D. Webster; (JAM, TEH, TUX)

8. U. meziana (Hitchc.) Morrone \& Zuloaga; (CUI, COX)

9. U. mollis (Sw.) Morrone \& Zuloaga; (JAM, JUC, TEH, TEO, TUX)

10. U. mutica (Forssk.) T. Q. Nguyen; (TUX)

11. U. reptans (L.) Stapf; (CUI, TEH, TUX)

12. U. plantaginea (Link) R. D. Webster; (TEO, ZAA)

Vulpia C. C. Gmel.; Pooideae; 2 especies.

1. V. bromoides (L.) Gray; registrada por Espejo et al. (2000). No se especifica la localidad dentro del estado.

2. V. myuros (L.) C. C. Gmel.; (CUI, ETL, HUA, JUX, IXT, MIA, TEP)

Zea L.; Panicoideae; 2 especies, una representada por 2 subespecies.

1. Z. luxurians (Durieu \& Asch.) Bird.; (TEH)

2. Z. mays L. subsp. mays; (CHO, COX, MIX, TUX, TLA)

2. Z. mays L. subsp. mexicana (Schrad.) H. H. Iltis; (COX, TUX)

Zeugites P. D. Brown; Centothecoideae; 4 especies, una representada por 2 variedades.

1. Z. americana Willd. var. mexicana (Kunth) McVaugh; (COX, JUX, TEH, TEO, TEP)

1. Z. americana Willd. var. pringlei (Scribn.) McVaugh; (JUX, MIA) 
Apéndice. Continuación.

2. Z. capillaris (A. Hitchc.) Swallen; (TEH)

3. Z. latifolia (Rupr. ex E. Fourn.) Hemsl.; registrada por Espejo et al. (2000). No se especifica la localidad dentro del estado.

4. Z. smilacifolia Scribn.; (JUX) 